\section{P1-396 HEPATITIS B VIRUS (HBV) INFECTION IN RELATION TO CONSISTENT CONDOM USE: A POPULATION-BASED STUDY IN PERU}

doi:10.1136/jech.2011.142976f.87

\begin{abstract}
${ }^{1} \mathrm{~A}$ Bernabe-Ortiz, ${ }^{1} \mathrm{C}$ Carcamo, ${ }^{2} \mathrm{~J}$ Scott, ${ }^{2} \mathrm{~J}$ Hughes, ${ }^{1} \mathrm{P}$ Garcia, ${ }^{2} \mathrm{~K}$ Holmes. ${ }^{1}$ Universidad Peruana Cayetano Heredia, Lima, Peru; ${ }^{2}$ University of Washington, Seattle, Washington, USA
\end{abstract}

Introduction Data on hepatitis B virus (HBV) are limited in developing countries. The study goal was to evaluate the prevalence and factors associated with HBV infection in Peru, highlighting its relationship with consistent condom use.

Methods Data from two different surveys performed in 28 midsized Peruvian cities were used. Participants aged 18-29 years were selected using a multistage cluster sampling. Information was collected through a validated two-part questionnaire. The first part (face-to-face) concerned demographic data, while the second part (self-administered using handheld computers) concerned sexual behaviour. Hepatitis B core antibody (anti-HBc) was tested in 7000 blood samples. Prevalences and associations were adjusted for sample strata, primary sampling units and population weights.

Results Anti-HBc prevalence was 5.0\% (95\% CI 4.1\% to 5.9\%), with the highest prevalence among jungle cities: $16.3 \%$ (95\% CI 13.8\% to $19.1 \%)$. Anti-HBc positivity was associated with geographic region (highlands $\mathrm{OR}=2.05 ; 95 \% \mathrm{CI} 1.28$ to 3.27 , and jungle $\mathrm{OR}=4.86 ; 95 \%$ CI 3.05 to 7.74; compared to coastal region); and age at sexual debut $(\mathrm{OR}=0.90 ; 95 \%$ CI 0.85 to 0.97$)$. Consistent condom use was associated with lower prevalence ( $\mathrm{OR}=0.34 ; 95 \% \mathrm{CI} 0.15$ to 0.79 ) after adjusting for sex, geographic region, education level, lifetime number of sex partners, and age at sexual debut.

Conclusion Residence in highlands or jungle cities is associated with higher anti-HBc prevalences, whereas increasing age at sexual debut was associated with lower prevalences. Consistent condom use was associated with decreased risk of anti-HBc. These findings emphasise the need of vaccination especially in jungle population, and imply that condom use promotion might be a strategy to prevent HBV infection

\section{P1-397 CHANGES IN UTILISATION OF HEALTH SERVICES AFTER REMOVAL OF USER FEES: OBSERVATIONS IN RURAL NEPAL}

doi:10.1136/jech.2011.142976f.88

\footnotetext{
${ }^{1,2}$ A Bhurtyal, ${ }^{*}{ }^{3}$ A K Poudyal, ${ }^{4}$ M K Maskey, ${ }^{3} \mathrm{D}$ Adhikari, ${ }^{1} \mathrm{D}$ R Sharma, ${ }^{5} \mathrm{~K}$ Bhurtyal. ${ }^{1}$ People's Health Initiative, Kathmandu, Nepal; ${ }^{2}$ World Health Organization, Country Office Nepal, Kathmandu, Nepal; ${ }^{3}$ Department of Community Medicine and Family Health, Institute of Medicine, Tribhuvan University, Kathmandu, Nepal; ${ }^{4} \mathrm{Nepal}$ Public Health Foundation, Kathmandu, Nepal; ${ }^{5}$ Independent medical doctor, New York, USA

Introduction Nepalese society is divided across hierarchical strata of gender, ethnicity and economic class, with women, ascribed "lower caste" and poor people placed at the bottom of society. Since 2007, the ministry of health and population removed user fees in primary health services in an attempt to increase use of the services, particularly among underserved population.

Methods Records of 1850 health services users were selected randomly from peripheral facilities-district hospital, primary healthcare centre, and 4 (of 8) health posts-of Jumla-a rural mountain district. Proportions of health services use by privileged and underprivileged ethnicities, men and women, and rich and poor people; before and after user fee removal, were calculated. Semistructured interviews were conducted among health services users, management staff, and representatives of donors and NGOs working in the district.
}

Results After fee removal, use of health services by women, underprivileged ethnicities, and poor people increased by $2.4 \%, 6.8 \%$ and $9.2 \%$ respectively. However, users and providers had conflicting opinions over the delivery of free health services. There was no evidence to establish the role of health system related confounders, however, it was suspected that ongoing process of state democratisation may have influenced the observed changes.

Conclusion Removal of user fees and subsequent increase in health services use by marginalised people as compared to their privileged counterparts is encouraging. However, some impediments still remain, which can be overcome by improving the delivery system with a focus on smoothening user-provider interactions.

\section{P1-398 VALIDATION OF A QUESTIONNAIRE TO ASSESS PHYSICAL ACTIVITY IN CHILDREN}

doi:10.1136/jech.2011.142976f.89

${ }^{1} \mathrm{R}$ Bielemann, ${ }^{2} \mathrm{~F}$ Reichert, ${ }^{3} \mathrm{~V}$ Paniz, ${ }^{1} \mathrm{D}$ Gigante. ${ }^{1}$ Post-Graduate Program in Epidemiology, Federal University of Pelotas, Pelotas, Rio Grande do Sul, Brazil; ${ }^{2}$ PostGraduate Program in Physical Education, Federal University of Pelotas, Pelotas, Rio Grande do Sul, Brazil; ${ }^{3}$ Post-Graduate Program in Population Health, University of Vale do Rio do Sinos, São Leopoldo, Rio Grande do Sul, Brazil

Background Subjective instruments for physical activity assessment in children have particularities, and to assess the reliability of these instruments is crucial. The aim of this study was to determine the validity of the Netherlands Physical Activity Questionnaire (NPAQ).

Methods Population under study was Brazilian children aged 4 to 11 years old. Data collection took place by application of the NPAO through face-to-face interviews with mothers children and utilisation of GT1M Actigraph accelerometers by five consecutive days as the reference method. Validity analyses were performed by correlation coefficient, sensibility and specificity, as well ROC curve.

Results Two hundred and thirty nine children participated of the study. The prevalence of physical activity was $73.2 \%$. The mean and median of the NPAO score were 25.5 and 26, respectively. Based on the area under the ROC curve, the median value presented the best indicators of sensibility (59.4\%) and specificity (60.9\%), and the area under curve was 0.63 . The predictive capacity of the NPAO to identify active children was high regardless the cut-off point chosen. This capacity was even higher from the score of 30 points on. The correlation coefficients between the last question of the instrument and the accelerometer variables were better than those from the rest of the NPAO.

Conclusions Based on sensibility and specificity values, the NPAO did not show satisfactory validity. More results of the predictive capacity of the last question will be better explored. Physical activity level of children estimated from questionnaires must be interpreted with cautions.

\section{P1-399 DATING VIOLENCE: PREVALENCE OF PHYSICAL VIOLENCE AND DIRECTIONALITY PATTERN IN TEN BRAZILIAN CITIES}

doi:10.1136/jech.2011.142976f.90

${ }^{1} \mathrm{M}$ L Lima, ${ }^{2} \mathrm{~S}$ Assis, ${ }^{3} \mathrm{~K}$ Njaine, ${ }^{4} \mathrm{~A}$ Kelly, ${ }^{5} \mathrm{M}$ Bigras. ${ }^{*}$ Centro de Pesquisas Aggeu Magalhães, Recife, Pernambuco, Nordeste, Brazil, ${ }^{2}$ Escola Nacional de Saúde PúblicaFIOCRUZ, Rio de Janeiro, Sudeste, Brazil; ${ }^{3}$ Escola Nacional de Saúde PúblicaFIOCRUZ, Brazil; " Universidade Federal de Pernambuco, Recife, Pernambuco, Nordeste, Brazil, ${ }^{5}$ Université du Québec à Montréal, Montréal, Québec, Sul, USA

The study investigates the prevalence and directionality pattern (only male, only female, or both are perpetrators) of physical 
violence in dating relationships perpetrated by secondary-school adolescents in 10 Brazilian cities. The hypothesis is that reciprocity pattern prevails and that female adolescents show the highest rates of perpetration. A sample of 3205 adolescents, aged 15-19, from state to private schools, was investigated, using the Conflict in Adolescent Dating Relationships Inventory. The majority of participants were female adolescents (59\%). The prevalence of violence perpetrated by male adolescents stood at $22.4 \%$, whereas for the female participants it was $39.4 \%$. The analysis of the directionality pattern shows that in most relationships both partners practiced aggression, corroborating the evidence for violence reciprocity. This pattern was found in four out of the ten cities, while in the other six, violence perpetrated by female adolescents reached the highest prevalence rates (average of $43.1 \%$ ). However, in all ten cities, violence perpetrated only by male adolescents shows lower prevalence levels, reaching an average of $9.7 \%$. In line with other studies in Brazil and the USA, female adolescents seem to be more violent than male teenagers, contradicting common sense and the findings from studies on violence among adult couples, in which case women are the main victims. Further investigation is needed into the following aspects: severity and frequency of violent acts; evolution of violence dynamics from dating to adult relationships; and the underlying reasons and context in which violence occurs.

\section{P1-400 INFANT MORTALITY FROM PREVENTABLE CAUSES IN BRAZIL: AN ECOLOGICAL STUDY}

doi:10.1136/jech.2011.142976f.91

A Boing, , A Boing. Federal University of Santa Catarina, Florianópolis, Santa Catarina, Brazil

Infant mortality is a major public health issue in developing countries. This study aims to test the associations between infant mortality from preventable causes in Brazil and socioeconomic factors, including those pertaining to health services and investments. This was an ecological study using data from 2000 to 2002. 296 Brazilian counties (municipalities) with more than 80000 inhabitants each were the analytical units. Kruskall-Wallis and ANOVA tests were performed to compare independent variables according to infant mortality quartile, and Pearson and Spearman's correlation coefficients were computed to test the associations. As the infant mortality quartile from preventable causes increases, there is a gradual decrease in the municipal human development index, per capita gross domestic product, households with bathrooms and indoor plumbing, total health expenditures per inhabitant, and physicians per 1000 inhabitants, and an increase in the Gini coefficient. Improved socioeconomic conditions and public health investments are strongly associated with reduction of infant mortality from preventable causes. This knowledge should permeate actions aimed at minimising the number and unequal distribution of such deaths.

\section{P1-401 TEMPORAL TREND IN AND SPATIAL DISTRIBUTION OF LUNG CANCER MORTALITY IN BRAZIL BETWEEN 1979 AND 2004: MAGNITUDE, REGIONAL PATTERNS, AND GENDER- RELATED DIFFERENCES}

doi:10.1136/jech.2011.142976f.92

A F Boing, ${ }^{*}$ T F Rossi. Federal University of Santa Catarina, Florianópolis, Santa Catarina, Brazil

Introduction Lung cancer is a major public health problem in Brazil. The aim of this study was to describe the temporal trend and the spatial distribution of mortality from tracheal, bronchial, and lung cancer in Brazil from 1979 to 2004.

Methods Mortality data by gender and geographic region were obtained from the Mortality Database created by the Ministry of Health in 1975. Demographic data were collected from the national censuses, from population counts, and from population estimates made in non-census years. Mortality rates were standardised according to the direct method, and the trends were analysed by gender and geographic region using the Prais-Winsten method for generalised linear regression.

Results Lung cancer mortality accounted for approximately $12 \%$ of the overall neoplasia-related mortality during the period. There was a trend towards an increase for both genders and in all regions, except for the male population in the southeast region, whose rates remained steady between 1979 and 2004 . The highest rates were observed in the south and southeast regions. However, the northeast region was the one that presented the greatest increase, followed by the central-west and north regions. In all regions, the increase in mortality rates was higher in women.

Conclusion The increase in lung cancer mortality in Brazil between 1979 and 2004 requires public measures that can minimise exposition to risk factors, mainly tobacco, and allow greater access to healthcare facilities for diagnosis and treatment.

\section{P1-402 PSYCHOSOCIAL PATHWAYS TO MENTAL WELL-BEING IN DEPRIVED AREAS}

doi:10.1136/jech.2011.142976f.93

${ }^{1} \mathrm{~L}$ Bond, ${ }^{* 1,2} \mathrm{~A}$ Kearns, ${ }^{1,2} \mathrm{P}$ Mason, ${ }^{1,3} \mathrm{C}$ Tannahill, ${ }^{1} \mathrm{M}$ Egan, ${ }^{1,2} \mathrm{E}$ Whitley. ${ }^{1} \mathrm{MRC} / \mathrm{CSO}$ Social \& Public Health Sciences Unit, Glasgow, UK; ${ }^{2}$ University of Glasgow, Glasgow, UK; ${ }^{3}$ Glasgow Centre for Population Health, Glasgow, UK

Introduction Housing-led regeneration has been shown to have limited effects on mental health. Considering neighbourhoods as a psychosocial environment, regeneration may have greater impact on mental well-being than mental ill-health. This study examined the relationship between the well-being of residents living in deprived areas and aspects of housing, neighbourhoods and communities.

Methods A cross-sectional study of 3911 residents in 15 deprived areas in Glasgow, Scotland. Mental well-being was measured using the Warwick Edinburgh Mental Well-being Scale.

Results Using multivariate analysis and controlling for sociodemographic characteristics and physical health status, we found that residential and environmental aspects of people's houses and neighbourhoods were strongly associated with wellbeing: when respondents considered that their neighbourhood had very good aesthetic qualities (RR 3.3, 95\% CI 1.9 to 5.8), their home and neighbourhood represented personal progress (RR 3.2 $95 \%$ CI 2.2 to 4.8 ; RR 2.6, 95\% CI 1.8 to 3.7 , respectively), they perceived their residence to have a very good external appearance (RR 2.6, 95\% CI 1.3 to 5.1); a very good front door (both an aesthetic and a security/control item) (RR 2.1, 95\% CI 1.2 to 3.8$)$, and satisfaction with their landlord was very high (RR 2.3, 95\% CI 2.2 to 4.8). Perception of poor neighbourhood aesthetic quality was associated with lower well-being (RR 0.4, 95\% CI 0.3 to 0.5 )

Discussion This study has found that where we live matters for mental well-being. In particular, it appears that positive residential circumstances may influence how we feel about ourselves and our view of our position in society, with beneficial consequences for well-being.

\section{P1-403 WITHDRAWN}

